\section{Turning back the malarial hordes}

\section{By Tim Fulmer, Senior Writer}

Researchers from the Wellcome Trust Sanger Institute have identified a host receptor, basigin Ok blood group, that allows Plasmodium falciparum to invade erythrocytes and trigger a blood-stage malaria infection. ${ }^{1}$ The researchers are now developing a vaccine to neutralize the $P$. falciparum antigen that binds the receptor. A vaccine targeting blood-stage infection could be more effective at preventing clinical symptoms of malaria than vaccines that target the asymptomatic liver stage of the parasite.

The life cycle of $P$. falciparum has three distinct stages. The liver and blood stages occur in the host, whereas the sexual stage occurs in the gut of the mosquito. The actual illness only occurs during blood-stage infection, which is triggered when the parasite is released from the liver, enters systemic circulation and invades erythrocytes.

Most malaria vaccines in development target liver-stage antigens because there is less antigenic variation at the liver stage than at the blood stage. ${ }^{2}$ Nonetheless, blood-stage vaccines remain an active area of research, with the goal of preventing clinical symptoms caused by any parasites that manage to escape from the liver and enter the blood.

The invasion process is complex and involves multiple parasite antigens that interact with proteins on the exterior and interior of the red blood cell. ${ }^{3}$

Thus, to prevent parasite invasion "it will be necessary either to identify key essential invasion pathways or to simultaneously target multiple invasion pathways, which would increase the complexity of the vaccine," said Joseph Smith, affiliate associate professor of global health at the University of Washington.

A team led by Julian Rayner and Gavin Wright hypothesized that P. falciparum reticulocyte-binding protein homolog 5 (PfRh5) might be the key antigen for invasion. Rayner is group leader in the Malaria Programme at Wellcome Trust. Wright is group leader of the cell surface signaling laboratory there.

The group was tipped off by a 2009 paper by Alan Cowman and colleagues that showed PfRh5 bound erythrocytes in vitro and, more importantly, was essential for growth in blood-stage cultures. ${ }^{4,5}$ Cowman is head of the division of infection and immunity at The Walter and Eliza Hall Institute of Medical Research (WEHI) and professor of medical biology at The University of Melbourne.
The Wellcome team reasoned that if PfRh5 was essential for invasion, the next step was identifying its binding partner on erythrocytes and then testing whether preventing PfRh5 from binding its receptor blocked invasion.

Using published proteomics data, ${ }^{6}$ the researchers first generated a library of 40 abundant cell surface and secreted proteins expressed by human erythrocytes. They then screened purified PfRh5 against that library using a method developed in Wright's lab called AVEXIS (avidity-based extracellular interaction screen), which is designed to detect transient extracellular protein-protein interactions. ${ }^{7}$

A single erythrocyte protein interacted strongly with PfRh5: basigin Ok blood group (BSG; EMMPRIN; CD147), a ubiquitously expressed membrane glycoprotein involved in gestation, spermatogenesis, retinal development and leukocyte activation.

Indeed, anti-BSG antibodies blocked erythrocyte invasion by multiple strains of $P$. falciparum compared with isotype controls. Also, small hairpin RNA against BSG in two $P$. falciparum strains decreased invasion compared with scrambled control shRNA.

The data, which were published in Nature, suggest that interactions between the host receptor BSG and the malarial ligand PfRh5 are critical for erythrocyte invasion.

"The hope is that a vaccine based around PfRh5 would induce antibodies that prevent parasites from invading red blood cells and therefore protect against the worst symptoms and complications of malaria," corresponding author Rayner told SciBX.

"The major attractions of targeting the BSG-PfRh5 interaction are threefold," added Jacob Baum. "First, there's the apparently essential nature of the interaction across all falciparum strains tested. Second, $\mathrm{PfRh} 5$ is a relatively small member of the Rh family of proteins, which means there's a real possibility of using the whole protein as a vaccine antigen. Third, PfRh5 seems to have very limited polymorphism, which could facilitate its universality as a vaccine candidate."

Baum did much of the original work on PfRh5 when he was a researcher in Cowman's lab. He is now laboratory head in the division of infection and immunity at WEHI and a senior research fellow at the University of Melbourne.

Low polymorphism could be an important feature of a PfRh5-based vaccine because "one of the main challenges for blood-stage vaccines has been polymorphism in parasite invasion ligands," said Smith. "AMA1 [ $P$. falciparum apical membrane antigen 1] is another parasite protein that plays a critical role in invasion. There has been significant work to make a vaccine against AMA1. The problem is that AMA1 is highly polymorphic, and anti-AMA1 antibodies are strain specific and do not confer broad protection."

Smith is also associate member and interim director of the malaria program at the Seattle Biomedical Research Institute.

Seattle Biomedical is developing live, attenuated malaria vaccines. In June, the institute published that a genetically modified malaria strain 


\section{ANALYSIS}

with attenuated growth at the liver stage was nonetheless able to induce a strong $\mathrm{T}$ cell response and protect mice from infection. ${ }^{8}$

\section{Moving toward a vaccine}

Rayner said his team has already begun to solve a key problem facing any vaccine program: antigen production. "If PfRh5 is to be used in a vaccine, we need to be able to produce it in large amounts. The system we used to produce PfRh5 in the paper is very efficient and may well be able to be scaled up to produce protein for testing in small clinical trials," he said.

Rayner declined to provide any additional details on next steps.

"It will be important to show whether recombinant $\mathrm{PfRh} 5$ proteins can elicit antibodies that inhibit parasite invasion and determine whether those antibodies inhibit different parasite strains," said Smith.

Although it is still early to compare a PfRh5-based blood-stage vaccine with the liver-stage vaccines in development, Rayner said, "PfRh5 could well turn out to be complementary to those approaches. By targeting two different stages of the life cycle, we may be able to generate better protection than by targeting either stage alone."

The most advanced malaria vaccine, RTS,S (Mosquirix) from GlaxoSmithKline plc, targets a key antigen of the liver stage: the circumsporozoite protein. In October, GSK published in The New England Journal of Medicine Phase III data showing that the vaccine lowered the incidence of clinical malaria by $55.8 \%$ and severe malaria by $47.3 \%$ in children aged $5-17$ months versus a nonmalaria comparator vaccine $\left(p<0.001\right.$ for both). ${ }^{9}$
Cowman told SciBX his lab is also working on a PfRh5-based malaria vaccine but declined to provide further details.

According to Rayner, the Nature findings are covered by a patent. He declined to disclose the licensing status of that IP.

Fulmer, T. SciBX 4(46); doi:10.1038/scibx.2011.1282

Published online Dec. 1, 2011

\section{REFERENCES}

1. Crosnier, C. et al. Nature; published online Nov. 9, 2011; doi:10.1038/nature10606

Contact: Julian Rayner, Wellcome Trust Sanger Institute, Cambridge, U.K.

e-mail: jr9@sanger.ac.uk

Contact: Gavin J. Wright, same affiliation as above e-mail: gw2@sanger.ac.uk

2. Richards, J.S. \& Beeson, J.G. Immunol. Cell Biol. 87, 377-390 (2009)

3. Iyer, J. et al. Mol. Microbiol. 65, 231-249 (2007)

4. Baum, J. et al. Int. J. Parasitol. 39, 371-380 (2009)

5. Triglia, T. et al. Cell. Microbiol. 11, 1671-1687 (2009)

6. Pasini, E.M. et al. Blood 108, 791-801 (2006)

7. Bushell, K.M. et al. Genome Res. 18, 622-630 (2008)

8. Butler, N.S. et al. Cell Host Microbe 9, 451-462 (2011)

9. The RTS,S Clinical Trials Partnership. N. Engl. J. Med. 365, 1863-1875 (2011)

COMPANIES AND INSTITUTIONS MENTIONED

GlaxoSmithKline plc (LSE:GSK; NYSE:GSK), London, U.K. Seattle Biomedical Research Institute, Seattle, Wash. The University of Melbourne, Melbourne, Victoria, Australia University of Washington, Seattle, Wash. The Walter and Eliza Hall Institute of Medical Research, Parkville, Victoria, Australia Wellcome Trust Sanger Institute, Cambridge, U.K. 